\title{
A Mathematical Model for the Dynamics of Cholera with Control Measures
}

\author{
Stephen Edward ${ }^{1, *}$, Nkuba Nyerere ${ }^{2}$ \\ ${ }^{1}$ Department of Mathematics, College of Natural and Mathematical Sciences, University of Dodoma, Dodoma, Tanzania \\ ${ }^{2}$ Departments of Biometry and Mathematics, Sokoine University Of Agriculture, Morogoro, Tanzania
}

Email address:

stephenmwaihuti@yahoo.com (S. Edward), emmankuba@suanet.ac.tz (N. Nyerere)

\section{To cite this article:}

Stephen Edward, Nkuba Nyerere. A Mathematical Model for the Dynamics of Cholera with Control Measures. Applied and Computational Mathematics. Vol. 4, No. 2, 2015, pp. 53-63. doi: 10.11648/j.acm.20150402.14

\begin{abstract}
Cholera, an acute gastro-intestinal infection and a waterborne disease continues to emerge in developing countries and remains an important global health challenge. In this paper, we formulate a mathematical model that captures some essential dynamics of cholera transmission with public health educational campaigns, vaccination, sanitation and treatment as control strategies in limiting the disease. The reproduction numbers with single and combined controls are computed and compared with each other to assess the possible community benefits. Numerical simulation shows that in a unique control strategy, treatment yields the best results followed by education campaign, then sanitation and vaccination being the last. Furthermore, we noted that the control of cholera is very much better when we incorporated more than one strategy, in two controls the results were better than one strategy, and in three control strategies the results were far better than in two control strategies. Further simulations with all four interventions showed the best results among all combinations attained before. We performed sensitivity analysis on the key parameters that drive the disease dynamics in order to determine their relative importance to disease transmission and prevalence.
\end{abstract}

Keywords: Modeling, Sensitivity, Sanitation, Education, Treatment, Vaccination, Epidemiology

\section{Introduction}

Cholera is an example of a bacterial disease whose primary mode of infection is indirect; which is caused when individuals ingest fecal-contaminated water containing the bacteria $V$. cholera [5].Transmission between humans and reservoirs of pathogens implies that disease transmission includes an indirect route other than human-to-human contact

The last few years have witnessed many cholera outbreaks in developing countries, including India (2007), Congo (2008), Iraq (2008), Zimbabwe (2008-2009), Vietnam (2009), Nigeria (2010), and Haiti (2010). In the year of 2010 alone, it is estimated that cholera affects 3-5 million people and causes 100,000-130,000 deaths in the world annually [1].

In essence, Cholera is an infection of the small intestine caused by the gram-negative bacterium, Vibrio cholera. Untreated individuals suffer severely from diarrhea and vomiting. The disease can cause a rapid dehydration and electrolyte imbalance, and can lead to death. Meanwhile, different transmission pathways are possible. For example, a cholera outbreak in a Singapore psychiatric hospital indicated that the direct human-to-human transmission was a driving force [2].

The dynamics of cholera involve multiple interactions between the human host, the pathogen, and the environment [3], which contribute to both direct human-to-human and indirect environment-to-human transmission pathways.

Due to its huge impact on public health, and social and economic development, cholera has been the subject of extensive studies in clinical, experimental and theoretical fields. It remains an important global cause of morbidity and mortality, capable of causing periodic epidemic disease [4].

Typical at-risk areas include peri-urban slums, where basic infrastructure is not available, as well as camps for internally displaced people or refugees, where minimum requirements of clean water and sanitation are not met. Outcomes behind the disruption of water and sanitation systems or the displacement of populations to inadequate and overcrowded camps can increase the risk of cholera transmission. Cholera remains a global threat to public health and a key indicator of lack of social development. Recently, the reemergence of cholera has been noted in parallel with the ever-increasing size of vulnerable populations living in unsanitary conditions [6]. 
Education, which is a key tool in disease control, is often overlooked [9]. It requires investment in people rather than in biomedical interventions, but it has the potential to lead to enormous benefits for relatively low cost. Conversely, a lack of information can have a severe impact on worsening the spread of the disease. Cholera-specific education includes advising people with symptoms to seek medical care promptly, and improving sanitation and hygienic practices [13]. Failures to provide health education can be traced to barriers at one of six sites: to be effective, messages have to (1) reach the intended audience, (2) gain attention, (3) be correctly understood, (4) be accepted, (5) result in changed behavior and (6) result in improvement in health [14]. During the 1994 cholera epidemic of Guinea-Bissau, health education demonstrated that local preventive rituals, radio and word-of-mouth communication were effective educational tools [13].

The first scientists to suggest disinfecting water with chlorine were Louis- Bernard Guyton de Morveau (in France) and William Cumberland Cruikshank (in England), both around the year 1800, as it was found that water that has been treated with chlorine is effective in preventing the spread of water-borne diseases[15]. However, disinfection by chlorination can be problematic in some circumstances. Chlorine can react with naturally occurring organic compounds found in the water supply to produce disinfection byproducts (DBPs) such as trihalomethanes and haloacetic acids. Due to the potential carcinogenicity of these compounds, drinking water regulations across the developed world require regular monitoring of the concentration of these compounds in the distribution systems of municipal water systems. The World Health Organization (WHO) has stated that risks to health from DBPs are extremely small in comparison with inadequate disinfection.

Understanding the fundamental mechanism in the disease transmission is crucial for effective prevention and intervention strategies against a cholera outbreak. To this effect, mathematical modeling provides a unique approach to gain basic insights into the dynamics of infectious diseases. Therefore, by exploring the potential effects of diseasecontrol strategies such as water chlorination, mathematical modeling can predict the dynamics of explosive epidemics often associated with cholera outbreaks.

In an effort to gain deeper understanding of the complex dynamics of cholera, several mathematical models have been published. For example, Codeço in 2001 proposed a model [16] that explicitly accounted for the environmental component, i.e., the $V$. cholera concentration in the water supply, into a regular SIR epidemiological model. The incidence (or, the infection force) was modeled by a logistic function to represent the saturation effect. Hartley, Morris and Smith [17] in 2006 extended Codeço's work to include a hyper infectious state of the pathogen, representing the "explosive" infectivity of freshly shed $V$. cholerae, based on the laboratory observations [18]. This model was rigorously analyzed in [19]. Joh, Wang, Weiss et al. [20] in 2009 Modified Code,co's model by a threshold pathogen density for infection with a careful discussion on humanenvironment contact and in-reservoir pathogen dynamics. More recently, Mukandavire et al. [21] proposed a model to study the 2008-2009 cholera outbreaks in Zimbabwe. The model explicitly considered both human-to-human and environment-to-human transmission pathways. The results in this work demonstrated the importance of the human-tohuman transmission in cholera epidemics, especially in such places as Zimbabwe, a landlocked country in the middle of Africa. Moreover, Tien and Earn [22] in 2010 published a water-borne disease model which also included the dual transmission pathways, with bilinear incidence rates employed for both the environment-to-human and human-tohuman infection routes. No saturation effect was considered in Tien and Earn's work. A rigorous global stability analysis was conducted in [23] for many of the aforementioned models. In addition, Neilan et al. [24] in 2010 modified the cholera model proposed by Hartley, Morris and Smith [17] and added several control measures into the model. They consequently analyzed the optimal intervention strategies and conducted numerical simulation based on their model. No human-to-human infection route is considered in this work.

Jing Wang [19] considered three types of controls: vaccination, therapeutic treatment (including hydration therapy, antibiotics, etc.), and water sanitation but he did not incorporate the role of education control strategy in their model, also they did not consider a logistic growth of vibrio cholera.

Therefore our objective is to modify the model of Jing Wang [19] by adding education parameter as a control strategy and also consider that vibrio bacteria grow logistically. So we have four types of controls: vaccination, therapeutic treatment (including hydration therapy, antibiotics, just to mention a few), water sanitation and education campaign. In general, these control measures are functions of time. For the special case with constant controls, we are able to rigorously analyze the stabilities of the corresponding autonomous dynamical system. We will then use numerical simulation to explore various optimal control solutions involving single and multiple controls.

The rest of the paper is organized as follows: Section 2, introduces the continuous model that captures all four control strategies, Section 3, will deal with deriving the basic reproduction number with all control strategies from which we derive other reproduction numbers basing on whether a strategy is unique, or a combination of two or more strategies. In Section 4, we perform numerical simulations of the reproduction numbers derived. In section 5, we perform sensitivity analysis. Section 6 will close up the paper with a conclusion and some recommendations.

\section{Model Formulation}

The cholera model developed in [4] is a combined system of human populations and the environmental component (SIR-B), with the environment-to-human transmission represented by a logistic (or Michaelis-Menten type) function 
and the human-to-human transmission by the standard mass action law. We now extend this model by adding vaccination, treatment, education campaign and water sanitation.

The total human population is divided into three compartments depending on the epidemiological status of individuals. These compartments include: Susceptible, $S(t)$, symptomatically infected, $I(t)$ and Recovered, $R(t)$. We assume that the total population is non-constant, which is a reasonable assumption for a relatively short period of time and for low-mortality diseases such as cholera. The concentration of the vibrios in the environment (that is contaminated water) is denoted by $B(t)$. Furthermore, the susceptible population increases due to the incoming of immigrants and recovered individuals at the rates $\Lambda$ and $\phi$ respectively. On the other hand, the susceptible population decreases due to the infection and vaccination strategy.

Concentration of Vibrio Cholerae in food and water that yields $50 \%$ chance of catching cholera disease $\mathrm{K}, \gamma$ is the rate at which infected people recovered from cholera disease, $w$ is the loss rate of Vibrio Cholerae in the environment. $\mathcal{E}$ is the contribution of each infected person to the population of Vibrio Cholerae in the environment.

\subsection{Model Assumptions}

The formulation of our model is guided by the following assumptions:

i. The total population of individuals is not constant.

ii. Controls are implemented continuously.

iii. Vaccination is introduced to the susceptible population.

iv. Therapeutic treatment is applied to the infected individuals.

v. Water sanitation leads to the death of vibrios.

vi. On recovery, there temporary immunity.

The above description leads to the compartmental diagram in Figure 1. The parameters indicated in Figure 1 are described in Table 1.

$\theta 9$

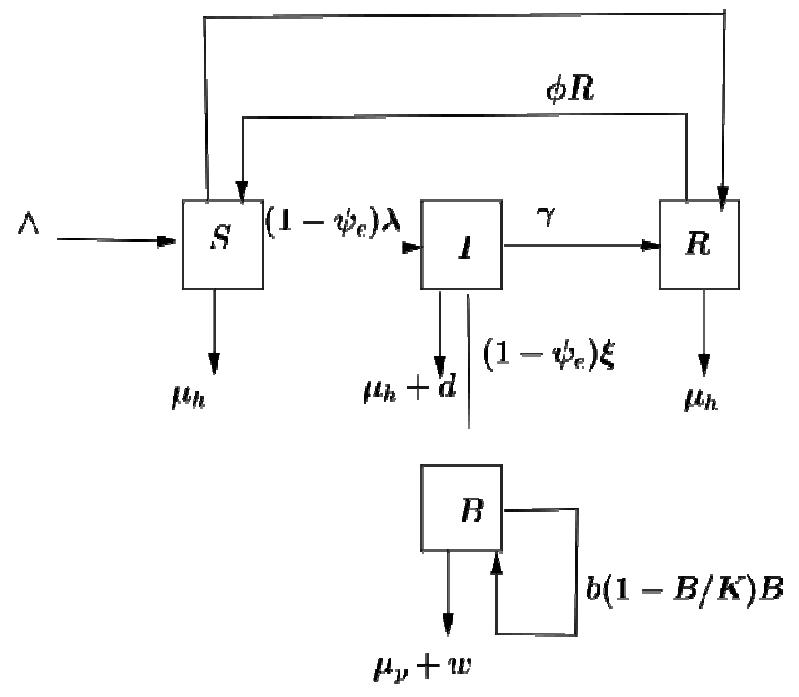

Figure 1. Compartmental diagram for a Cholera Model with control strategies
Table 1. Parameters and their description.

\begin{tabular}{ll}
\hline Parameter & Sensitivity Index \\
\hline$\Lambda$ & Constant human recruitment rate \\
$\mu_{p}$ & Mortality rate for bacteria, including phage degradation \\
$\mu_{h}$ & Natural human mortality rate \\
$d$ & Disease induced death rate \\
$\alpha_{2}$ & Effective contact rate between individuals (contact \\
$\alpha_{1}$ & sufficient) \\
$\gamma$ & Per capita contact rate for humans and contaminated water \\
$\varepsilon$ & Recovery rate of infected humans \\
$\phi$ & Bacteria shed rate into the water supply by infected human \\
$\theta$ & Per capita rate at which recovered humans are susceptible \\
$1-\psi_{e}$ & Per capita rate at which susceptible humans are vaccinated \\
$b$ & Education parameter \\
$K$ & (Maximum) per capita growth rate for $V$. cholerae bacteria \\
$w$ & Carrying capacity for $V$. cholerae \\
\hline
\end{tabular}

\subsection{The Model Equations}

From the assumptions, descriptions and the compartmental diagram in Figure 1, we formulate the following system of differential equations.

$$
\begin{gathered}
\frac{d S}{d t}=\Lambda+\phi R-\left(1-\psi_{e}\right) \lambda S-\left(\mu_{h}+\theta\right) S \\
\frac{d I}{d t}=\left(1-\psi_{e}\right) \lambda S-\left(\mu_{h}+d+\gamma\right) I \\
\frac{d B}{d t}=b\left(1-\frac{B}{K}\right) B+\left(1-\psi_{e}\right) \varepsilon I-\left(\mu_{p}+w\right) B \\
\frac{d R}{d t}=\gamma I+\theta S-\left(\mu_{h}+\phi\right) R
\end{gathered}
$$

Equation (1) describes the dynamics of susceptible in the community of size $N$. The death rate of the susceptible individuals is represented by $\mu_{h}, \frac{\alpha_{1} B}{K+B}$ is the rate of recruitment into susceptible class, $\alpha_{1}$ is the rate of exposure to contaminated food and water, $\frac{\alpha_{1} B}{K+B}$ is the probability of susceptible catching cholera. $S$ is the susceptible and $B$ is the concentration of toxigenic Vibrio Cholerae bacteria in food and water.

Equation (2) describes the dynamics of infected people in the community, their number increases as susceptible become infected and decreases as the infected recovers or die from the disease or natural death. Measures to limit the spread of the disease, such as hygiene and total sanitation reduce the amount of Vibrio Cholerae bacteria in the environment.

Equation (3) describes the dynamics of pathogenic Vibrio Cholerae in the environment, comprising the contaminated food or water consumed by people and unhygienic handling of cholera patients and their waste products.

Equation (4) describes the dynamics of effect of treatment or lack of treatment to the population of infected people. 


\subsection{Basic Properties of the Model}

\subsubsection{Positivity of Solutions}

Since the model monitors human population, we need to show that all the state variables remain non-negative for all times.

Theorem 1

Let $\quad T=\left\{S, I, R, B \in \mathbb{R}^{4}{ }_{+}: S(0)>0, I(0)>0, R(0)>\right.$ $0, B(0)>0\}$ then the solutions of $\{S(t), I(t), R(t), B(t)\}$ of the system equation (1-4) are positive for all $t \geq 0$.

Proof:

Consider the $1^{\text {st }}$ equation of the system eqn. (1-4)

$$
\begin{gathered}
\frac{d S}{d t}=\Lambda+\phi R-\left(1-\psi_{e}\right) \lambda S-\left(\mu_{h}+\theta\right) S \\
\frac{d S}{d t} \geq \Lambda-\left(\mu_{h}+\theta\right) S \\
\frac{d S}{d t}+\left(\mu_{h}+\theta\right) S=\Lambda
\end{gathered}
$$

Solving we get

$$
S(t)=\frac{\Lambda}{\mu_{h}+\theta}+c e^{-\left(\mu_{h}+\theta\right) t}
$$

Taking initial conditions we get:

$$
S(t)=\frac{\Lambda}{\mu_{h}+\theta}+\left(s_{0}-\frac{\Lambda}{\mu_{h}+\theta}\right) e^{-\left(\mu_{h}+\theta\right) t} \geq 0
$$

Consider also the $2^{\text {nd }}$ equation of the system eqn. (1-4)

$$
\begin{gathered}
\frac{d I}{d t}=\left(1-\psi_{e}\right) \lambda S-\left(\mu_{h}+d+\gamma\right) I \\
\frac{d I}{d t} \geq-\left(\mu_{h}+d+\gamma\right) I
\end{gathered}
$$

Upon integration we get

$$
I(t)=M e^{-\left(\mu_{h}+d+\gamma\right) t}
$$

Taking initial conditions we get

$$
I(t) \geq I_{0} e^{-\left(\mu_{h}+d+\gamma\right) t} \geq 0
$$

Consider also the $3^{\text {rd }}$ equation of the system eqn. (1-4)

$$
\begin{gathered}
\frac{d B}{d t}=b\left(1-\frac{B}{K}\right) B+\left(1-\psi_{e}\right) \varepsilon I-\left(\mu_{p}+w\right) B \\
\frac{d B}{d t} \geq b\left(1-\frac{B}{K}\right) B-\left(\mu_{p}+w\right) B \\
\frac{d B}{d t}=b\left(1-\frac{B}{K}\right) B-\left(\mu_{p}+w\right) B
\end{gathered}
$$

$$
\frac{d B}{\frac{B^{2}}{K}+\left(\mu_{p}+w-b\right)}=-d t
$$

Upon integration we get

$$
B(t)=\frac{a_{2} f_{1} e^{-a_{2} t}}{1-a_{1} f_{1} e^{-a_{2} t}}
$$

Taking initial conditions we get

$$
\begin{aligned}
& B(t)=\frac{B_{0} a_{2} e^{-a_{2} t}}{B_{0} a_{1}\left(1-e^{-a_{2} t}\right)+a_{2}} \\
& \text { As } t \rightarrow+\infty \text { then } B \rightarrow 0
\end{aligned}
$$

Consider also the $3^{\text {rd }}$ equation of the system eqn. (1-4)

$$
\begin{gathered}
\frac{d R}{d t}=\gamma I+\theta S-\left(\mu_{h}+\phi\right) R \\
\frac{d R}{d t} \geq-\left(\mu_{h}+\phi\right) R \\
\frac{d R}{d t}=-\left(\mu_{h}+\phi\right) R
\end{gathered}
$$

Upon integration we get

$$
R(t)=C e^{-\left(\mu_{h}+\phi\right) t} \geq 0
$$

Taking initial conditions we get

$$
R(t)=R_{0} e^{-\left(\mu_{h}+\phi\right) t} \geq 0
$$

\subsubsection{Invariant Region}

The system equations (1-4) have solutions which are contained in the feasible region:

$$
T=\left\{(S, I, R, B) \in R_{+}^{4}: 0 \leq S, 0 \leq I, 0 \leq R, 0 \leq B, S+I+R \leq \frac{\Lambda}{\mu_{h}}\right\}
$$

where the model makes biological sense can be shown to be positively invariant and globally attracting in $R^{4}{ }_{+}$with respect to our system.

\section{Model Analysis}

The model system (1-4) is analyzed qualitatively to get insights into its dynamical features which give better understanding of the impact control strategies on the transmission dynamics of vibrio cholera virus.

\subsection{Disease Free Equilibrium (DFE), $P_{0}$}

The disease free equilibrium of model system (1-4) is obtained by setting $\frac{d S}{d t}=\frac{d I}{d t}=\frac{d R}{d t}=\frac{d B}{d t}=0$, and in the absence of disease, $\mathrm{I}=\mathrm{B}=0$ so that: 


$$
\begin{gathered}
S^{0}=\frac{\left(\mu_{h}+\theta\right) \Lambda}{\mu_{h}\left(\mu_{h}+\theta+\phi\right)} \\
R^{0}=\frac{\theta \Lambda}{\mu_{h}\left(\mu_{h}+\theta+\phi\right)}
\end{gathered}
$$

Hence DFE is

$$
\left(S^{0}, I^{0}, B^{0}, R^{0}\right)=\left(\frac{\left.\mu_{h}+\theta\right) \Lambda}{\mu_{h}\left(\mu_{h}+\theta+\phi\right)}, 0,0, \frac{\theta \Lambda}{\mu_{h}\left(\mu_{h}+\theta+\phi\right)}\right)
$$

\subsection{The Basic Reproduction Number, $R_{0}$}

The basic reproduction number denoted by $R_{0}$ is the average number of secondary infections caused by an infectious individual during his or her entire period of infectiousness (Diekmann et al) [25]. The basic reproduction number is an important non-dimensional quantity in epidemiology as it sets the threshold in the study of a disease both for predicting its outbreak and for evaluating its control strategies. Thus, whether a disease becomes persistent or dies out in a community depends on the value of the reproduction number, $R_{0}$. Furthermore, stability of equilibria can be analyzed using $R_{0}$. If $R_{0}<1$ it means that every infectious individual will cause less than one secondary infection and hence the disease will die out and when $R_{0}>1$, every infectious individual will cause more than one secondary infection and hence the disease will invade the population. A large number of $R_{0}$ may indicate the possibility of a major epidemic. For the case of a model with a single infected class, $R_{0}$ is simply the product of the infection rate and the mean duration of the infection.

In more complicated epidemics we compute the basic reproduction number, $R_{0}$ using the next generation operator approach by van den Driessche and Watmough [26].

We calculate the basic reproduction number by using the next generation operator method on the system eqn. (1-4).

The basic reproduction number is obtained by taking the largest (dominant) eigenvalue (spectral radius) of

$$
\mathrm{FV}^{-1}=\left[\frac{\partial \mathcal{F}_{i}\left(E_{0}\right)}{\partial x_{j}}\right]\left[\frac{\partial \mathcal{V}_{i}\left(E_{0}\right)}{\partial x_{j}}\right]^{-1}
$$$$
\mathrm{FV}^{-1}=\left[\begin{array}{cc}
\frac{\left(1-\psi_{e}\right) \alpha_{2} S^{0}}{\mu_{h}+d+\gamma}+\frac{\left(1-\psi_{e}\right)^{2} \alpha_{1} \varepsilon S^{0}}{\left(\mu_{h}+d+\gamma\right)\left(\mu_{p}+\omega-b\right) K} & \frac{\left(1-\psi_{e}\right) \alpha_{1}}{\left(\mu_{p}+\omega-b\right) K} \\
0
\end{array}\right]
$$

$$
R_{0}^{c}=\frac{\Lambda\left(\mu_{h}+\theta\right)\left(1-\psi_{e}\right)\left[\left(1-\psi_{e}\right) \alpha_{1} \varepsilon+\left(\mu_{p}+\omega-b\right) \alpha_{2} K\right]}{\mu_{h}\left(\mu_{h}+\theta+\phi\right)\left(\mu_{h}+d+\gamma\right)\left(\mu_{p}+\omega-b\right) K}
$$

where $\mathcal{F}_{i}$ is the rate of appearance of new infection in compartment $i, \mathcal{V}_{i}$ is the transfer of infections from one compartment $i$ to another and $E_{0}$ is the disease-free equilibrium.

From system equation of the system (1-4), we re-write the equations with infectious classes, $I$ and $B$. This leads to the system

$$
\begin{gathered}
\frac{d I}{d t}=\left(1-\psi_{e}\right) \alpha_{1} \frac{B S}{K+B}+\left(1-\psi_{e}\right) \alpha_{2} I S-\left(\mu_{h}+d+\gamma\right) I \\
\frac{d B}{d t}=\left(1-\psi_{e}\right) \varepsilon I-\left(\mu_{p}+w\right) B
\end{gathered}
$$

From system (7-8),

$$
\mathcal{F}_{i}=\left[\begin{array}{c}
\left(1-\psi_{e}\right) \frac{\alpha_{1} B S}{K+B}+\left(1-\psi_{e}\right) \alpha_{2} I S \\
0
\end{array}\right]
$$

Partial differentiation of $\mathcal{F}_{i}$ with respect to $I$ and $B$ gives:

$$
\mathrm{F}=\left[\begin{array}{cc}
\left(1-\psi_{e}\right) \alpha_{2} S^{0} & \frac{\left(1-\psi_{e}\right) \alpha_{1}}{K} S^{0} \\
0 & 0
\end{array}\right]
$$

On the other hand,

$$
\mathcal{V}_{i}=\left[\begin{array}{c}
\left(\mu_{h}+d+\omega\right) I \\
-b\left(1-\frac{B}{K}\right) B-\left(1-\psi_{e}\right) \varepsilon I+\left(\mu_{p}+\omega-b\right) B
\end{array}\right]
$$

Partial differentiation of $\mathcal{V}_{i}$ with respect to $I$ and $B$ gives:

$$
\mathrm{V}=\left[\begin{array}{cc}
\mu_{h}+d+\gamma & 0 \\
-\left(1-\psi_{e}\right) \varepsilon & \mu_{p}+\omega-b
\end{array}\right]
$$

It follows that:

$$
\mathrm{V}^{-1}=\left[\begin{array}{cc}
\frac{1}{\mu_{h}+d+\gamma} & 0 \\
\frac{-\left(1-\psi_{e}\right) \varepsilon}{\left(\mu_{h}+d+\gamma\right)\left(\mu_{p}+\omega-b\right)} & \frac{1}{\mu_{p}+\omega-b}
\end{array}\right]
$$

Thus,

Thus $R_{0}^{c}$, is the effective reproduction number (basic reproduction number with controls).

When there is no any intervention we have: $\theta=\omega=\psi_{e}=$ $\gamma=0$.

Thus, the basic reproduction number for system (1-4) is:

$$
R_{0}=\frac{\Lambda\left[\alpha_{1} \varepsilon+\left(\mu_{p}-b\right) \alpha_{2} K\right]}{\mu_{h}\left(\mu_{h}+d\right)\left(\mu_{p}-b\right) K}
$$




\subsubsection{Analysis of $R_{0}^{c}$ with Unique Control Strategy}

In this section, we use effective reproduction number in equation (10) to compute reproduction numbers for individual control strategy (intervention). The similar approach was done by Stephen [27, 28] and Nyerere [29, 30].

If vaccination is the only control that is $\theta \neq 0, \omega=\psi_{e}=$ $\gamma=0$ then the basic reproduction number with vaccination only is given by:

$$
R_{v}=\frac{\Lambda\left(\mu_{h}+\theta\right)\left[\alpha_{1} \varepsilon+\left(\mu_{p}-b\right) \alpha_{2} K\right]}{\mu_{h}\left(\mu_{h}+\theta+\phi\right)\left(\mu_{h}+d\right)\left(\mu_{p}-b\right) K}
$$

If education is the only control that is $\psi_{e} \neq 0, \theta=\omega=$ $\gamma=0$ then the basic reproduction number with vaccination only is given by:

$$
R_{e d}=\frac{\Lambda\left(1-\psi_{e}\right)\left[\left(1-\psi_{e}\right) \alpha_{1} \varepsilon+\left(\mu_{p}-b\right) \alpha_{2} K\right]}{\mu_{h}\left(\mu_{h}+d\right)\left(\mu_{p}-b\right) K}
$$

If treatment is the only control, we have $\gamma \neq 0, \theta=\omega=$ $\psi_{e}=0$ then the basic reproduction number with treatment only is given by:

$$
R_{T}=\frac{\Lambda\left[\alpha_{1} \varepsilon+\left(\mu_{p}-b\right) \alpha_{2} K\right]}{\left(\mu_{h}+\phi\right)\left(\mu_{h}+d+\gamma\right)\left(\mu_{p}-b\right) K}
$$

If sanitation is the only control that is $\omega \neq 0, \theta=\gamma=$ $\psi_{e}=0$ then the basic reproduction number with sanitation only is given by:

$$
R_{s a}=\frac{\Lambda\left[\alpha_{1} \varepsilon+\left(\mu_{p}+\omega-b\right) \alpha_{2} K\right]}{\mu_{h}\left(\mu_{h}+d\right)\left(\mu_{p}+\omega-b\right) K}
$$

\subsubsection{Analysis of $R_{0}^{c}$ with Two Control Strategies}

In this section, we further analyze the effective reproduction number in equation (10) by computing reproduction numbers for the combination of two control strategies (interventions).If the combination of vaccination and education campaign is the only intervention that is $\psi_{e} \neq 0, \theta \neq 0, \gamma=\omega=0$ then the basic reproduction number with vaccination and education campaign only is given by:

$$
R_{\text {ved }}=\frac{\Lambda\left(\mu_{h}+\theta\right)\left(1-\psi_{e}\right)\left[\left(1-\psi_{e}\right) \alpha_{1} \varepsilon+\left(\mu_{p}-b\right) \alpha_{2} K\right]}{\mu_{h}\left(\mu_{h}+\theta+\phi\right)\left(\mu_{h}+d\right)\left(\mu_{p}-b\right) K}
$$

If the combination of vaccination and treatment is the only intervention that is $\gamma \neq 0, \theta \neq 0, \psi_{e}=\omega=0$ then the basic reproduction number is given by:

$$
R_{v T}=\frac{\Lambda\left(\mu_{h}+\theta\right)\left[\alpha_{1} \varepsilon+\left(\mu_{p}-b\right) \alpha_{2} K\right]}{\mu_{h}\left(\mu_{h}+\theta+\phi\right)\left(\mu_{h}+d+\gamma\right)\left(\mu_{p}-b\right) K}
$$

If the combination of vaccination and water sanitation is the only intervention that is $\omega \neq 0, \theta \neq 0, \psi_{e}=\gamma=0$ then the basic reproduction number is given by:

$$
R_{0}^{c}=\frac{\Lambda\left(\mu_{h}+\theta\right)\left[\alpha_{1} \varepsilon+\left(\mu_{p}+\omega-b\right) \alpha_{2} K\right]}{\mu_{h}\left(\mu_{h}+\theta+\phi\right)\left(\mu_{h}+d\right)\left(\mu_{p}+\omega-b\right) K}
$$

If the combination of treatment and education campaign is the only intervention that is $\psi_{e} \neq 0, \gamma \neq 0, \omega=\theta=0$ then the basic reproduction number is given by:

$$
R_{T e d}=\frac{\Lambda\left(1-\psi_{e}\right)\left[\left(1-\psi_{e}\right) \alpha_{1} \varepsilon+\left(\mu_{p}-b\right) \alpha_{2} K\right]}{\left(\mu_{h}+\phi\right)\left(\mu_{h}+d+\gamma\right)\left(\mu_{p}-b\right) K}
$$

If the combination of water sanitation and education campaign is the only intervention that is $\psi_{e} \neq 0, \omega \neq 0, \gamma=$ $\theta=0$ then the basic reproduction number is given by:

$$
R_{\text {saed }}=\frac{\Lambda\left(1-\psi_{e}\right)\left[\left(1-\psi_{e}\right) \alpha_{1} \varepsilon+\left(\mu_{p}+\omega-b\right) \alpha_{2} K\right]}{\mu_{h}\left(\mu_{h}+d\right)\left(\mu_{p}+\omega-b\right) K}
$$

If the combination of water sanitation and treatment is the only intervention that is $\gamma \neq 0, \omega \neq 0, \psi_{e}=\theta=0$ then the basic reproduction number is given by:

$$
R_{\text {saT }}=\frac{\Lambda\left[\alpha_{1} \varepsilon+\left(\mu_{p}+\omega-b\right) \alpha_{2} K\right]}{\left(\mu_{h}+\phi\right)\left(\mu_{h}+d+\gamma\right)\left(\mu_{p}+\omega-b\right) K}
$$

\subsubsection{Analysis of $R_{0}^{c}$ with Three Control Strategies}

Lastly, we analyze the effective reproduction number in (10) by computing reproduction numbers for the combination of three control strategies (interventions).

If the combination of water sanitation, treatment and education campaign is the only intervention that is $\psi_{e} \neq$ $0, \omega \neq 0, \gamma \neq 0, \theta=0$ then the basic reproduction number is given by:

$$
R_{\text {saTed }}=\frac{\Lambda\left(1-\psi_{e}\right)\left[\left(1-\psi_{e}\right) \alpha_{1} \varepsilon+\left(\mu_{p}+\omega-b\right) \alpha_{2} K\right]}{\left(\mu_{h}+\phi\right)\left(\mu_{h}+d+\gamma\right)\left(\mu_{p}+\omega-b\right) K}
$$

If the combination of water sanitation, vaccination and education campaign is the only intervention that is $\theta \neq$ $0, \psi_{e} \neq 0, \omega \neq 0, \gamma=0$ then the basic reproduction number is given by:

$$
R_{\text {vsaed }}=\frac{\Lambda\left(\mu_{h}+\theta\right)\left(1-\psi_{e}\right)\left[\left(1-\psi_{e}\right) \alpha_{1} \varepsilon+\left(\mu_{p}+\omega-b\right) \alpha_{2} K\right]}{\mu_{h}\left(\mu_{h}+\theta+\phi\right)\left(\mu_{h}+d\right)\left(\mu_{p}+\omega-b\right) K}
$$

If the combination of treatment, vaccination and education campaign is the only intervention that is $\theta \neq 0, \psi_{e} \neq 0, \gamma \neq$ $0, \omega=0$ then the basic reproduction number is given by:

$$
R_{v T e d}=\frac{\Lambda\left(\mu_{h}+\theta\right)\left(1-\psi_{e}\right)\left[\left(1-\psi_{e}\right) \alpha_{1} \varepsilon+\left(\mu_{p}-b\right) \alpha_{2} K\right]}{\mu_{h}\left(\mu_{h}+\theta+\phi\right)\left(\mu_{h}+d+\gamma\right)\left(\mu_{p}-b\right) K}
$$

If the combination of treatment, vaccination and water sanitation is the only intervention that is $\theta \neq 0, \gamma \neq 0, \omega \neq$ $0, \psi_{e}=0$ then the basic reproduction number is given by:

$$
R_{v s a T}=\frac{\Lambda\left(\mu_{h}+\theta\right)\left[\alpha_{1} \varepsilon+\left(\mu_{p}+\omega-b\right) \alpha_{2} K\right]}{\mu_{h}\left(\mu_{h}+\theta+\phi\right)\left(\mu_{h}+d+\gamma\right)\left(\mu_{p}+\omega-b\right) K}
$$

\subsection{Local Stability of the Disease-Free Equilibrium}

Here, we investigate the local stability of the disease-free equilibrium point, $P_{0}\left(S^{0}, I^{0}, B^{0}, R^{0}\right)$.Thus, we linearize model system (1-4) by computing its Jacobian matrix, $J_{E}$. The Jacobian matrix is computed by differentiating each equation in the system with respect to the state variables $S, I, B, R$. 


$$
\begin{gathered}
J\left(E_{0}\right)=\left[\begin{array}{cccc}
-\left(\mu_{h}+\theta\right) & -b S^{0} & \frac{-a S^{0}}{K} & \phi \\
0 & b S^{0}-c & \frac{a S^{0}}{K} & 0 \\
0 & f & -g & 0 \\
\theta & \gamma & 0 & -\left(\mu_{h}+\phi\right)
\end{array}\right] \\
\operatorname{det}\left(J\left(E_{0}\right)\right)=-\left(\mu_{h}+\theta\right) \mid \begin{array}{r}
\operatorname{If} c-b S^{0}>0 \text { then } \operatorname{tr}(A)<0 \text { so we only need to show that } \\
\operatorname{det}(A)>0
\end{array} \\
=\left(\mu_{h}+\theta\right)\left(\mu_{h}+\phi\right)\left[g\left(c-b S^{0}\right)-\frac{f S^{0} a}{K}\right]-\theta \phi\left[g\left(c-b S^{0}\right)-\frac{f S^{0} a}{K}\right]=\left(g\left(c-b S^{0}\right)-\frac{f S^{0} a}{K}\right)\left(\left(\mu_{h}+\theta\right)\left(\mu_{h}+\phi\right)-\theta \phi\right) \\
\gamma
\end{gathered}
$$

Since all parameters are assumed positive then Consider the term $\left(\left(\mu_{h}+\theta\right)\left(\mu_{h}+\phi\right)-\theta \phi\right)=\mu_{h}\left(\mu_{h}+\theta+\phi\right)>0$

$$
\left(g\left(c-b S^{0}\right)-\frac{f S^{0} a}{K}\right)=g c-\left(b g S^{0}+\frac{f S^{0} a}{K}\right)=g c\left(1-\frac{S^{0}}{K g c}(K b g+f a)\right)=g c\left(1-\frac{1}{g c}\left(b g S^{0}+\frac{f S^{0} a}{K}\right)\right)=g c\left(1-R_{0}^{c}\right)
$$

Hence we have:

$$
\left[g\left(c-b S^{0}\right)-\frac{f S^{0} a}{K}\right]\left[\left(\mu_{h}+\theta\right)\left(\mu_{h}+\phi\right)-\theta \phi\right]=\left(\left(\mu_{h}+\theta\right)\left(\mu_{h}+\phi\right)-\theta \phi\right) g c\left(1-R_{0}\right)>0
$$

Thus $\operatorname{det}\left(J\left(E_{0}\right)\right)>0$ provided $R_{0}^{c}<1$, therefore the DFE is locally asymptotically stable.

\section{Simulation and Discussion}

An SIR-B model was formulated and analyzed. The main objective of this study was to assess the impact of the incorporated control strategies on the transmission dynamics of the disease. Numerical simulations of model system (1-4) are carried out using a set of parameter values given in Table 2. Matlab is used in the numerical simulations.

Graphical representations showing the variations in reproduction numbers with respect to contact rate between the susceptible and the contaminated environment are provided in Figures 2-4. Since most of the parameters values were not readily available; we used data from literature and the missing data were estimated. Table 2 shows the set of parameter values which were used.

Table 2. Parameters values used in model simulations.

\begin{tabular}{lll}
\hline Parameters & Value & Source \\
\hline$\theta$ & $0.4 /$ year $^{-1}$ & Estimated \\
$\phi$ & 0.1 days $^{-1}$ & Estimated \\
$\alpha_{1}$ & variable & Estimated \\
$\alpha_{2}$ & 0.00005 day $^{-1}$ & Mukandavire[33] \\
$\omega$ & $0.0005 /$ day $^{-1}$ & Estimated \\
$\gamma$ & 0.2 days $^{-1}$ & Hartley[34] \\
\hline
\end{tabular}

\begin{tabular}{lll}
\hline Parameters & Value & Source \\
\hline$\psi_{e}$ & $0.4 /$ day [0-1] & Mwasa [38] \\
$k$ & 100000 cells/ml & Estimated \\
$\Lambda$ & $10 /$ day & Musekwa[35] \\
$\mu_{h}$ & $0.022 /$ year & WHO[36] \\
$\mu_{p}$ & 0.02 days $^{-1}$ & Estimated \\
$d$ & $0.015 /$ day $^{-1}$ & WHO [37] \\
$b$ & $0.01 /$ year [0-1] & Mwasa [38] \\
$\mathcal{E}$ & $0.5\left(\right.$ cell/ml day ${ }^{-1}$ person $^{-1}$ & Estimated \\
\hline
\end{tabular}

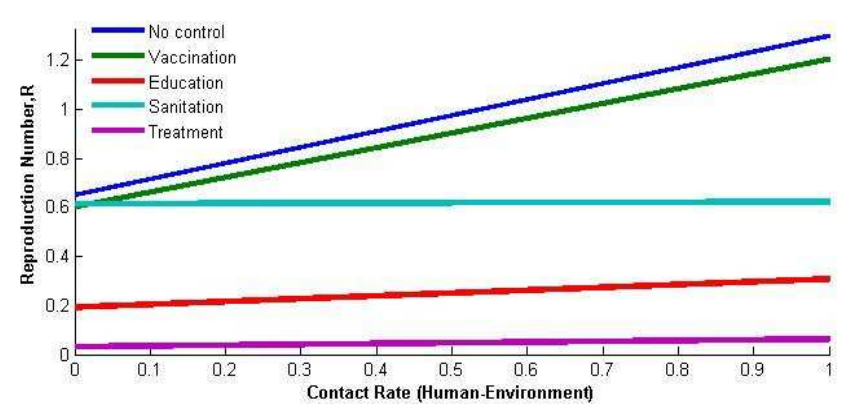

Figure 2.Variations of a mono-control reproduction number with respect to human-environment contact rate

Figure 2 shows that, $R_{e d}<\mathrm{R}_{T}<R_{s a}<R_{v}<R_{0}$. We see from the figure that $R_{0}$ is worst case scenario, it occurs when there is no any control strategy for the epidemic. The basic reproduction number $R_{0}$ grows very sharp with respect to an increase in human-environment contact rate. Such an 
increase in $R_{0}$ above unity implies that there is a high eruption of cholera in the community.

The best case scenario occurs at graph $\mathrm{R}_{T}$, here treatment is the only intervention offered to infected individuals. It can be noticed that the reproduction number with treatment strategy is very small indeed less than unity, which means that the disease dies out. After treatment individuals recover, since recovery is not permanent the recovered individuals might become susceptible again to the disease.

The next to best case scenario occurs at graph $\mathrm{R}_{\mathrm{e} d}$, here education campaign is emphasized to individuals. This includes education on self hygiene, importance of using toilets, drinking boiled water, humans not to contaminate water, use of oral salts to help already infected individuals, avoiding direct contact to infected individuals etc. It can be noticed from Figure 2 that education campaign is more important as compared to sanitation and vaccination because individual awareness about the disease limit the spread of the epidemic better.

The next to the best case scenario is $\mathrm{R}_{s a}$ which takes account for water sanitation. It is noted that treating water with chlorine plays an important role in combating cholera as compared to vaccination. This is because addition of chlorine in water kills the vibrio cholera virus whereas vaccinating individual just boost immunity of an individual, it is clear from literature that no vaccine is perfect, the vaccines usually wanes with time and thus previously vaccinated individual might be easily infected with the disease if the vaccine has already expired [31]. Another concern about vaccination is coverage; it is practically not easy to attain mass vaccination because of several reasons; including financial constraints and infrastructure constraints. That is why many governments opt to offer clean water to its individuals because it is not only cheaper but also is healthier than vaccination. In so doing they tend to limit the eruption of the disease.

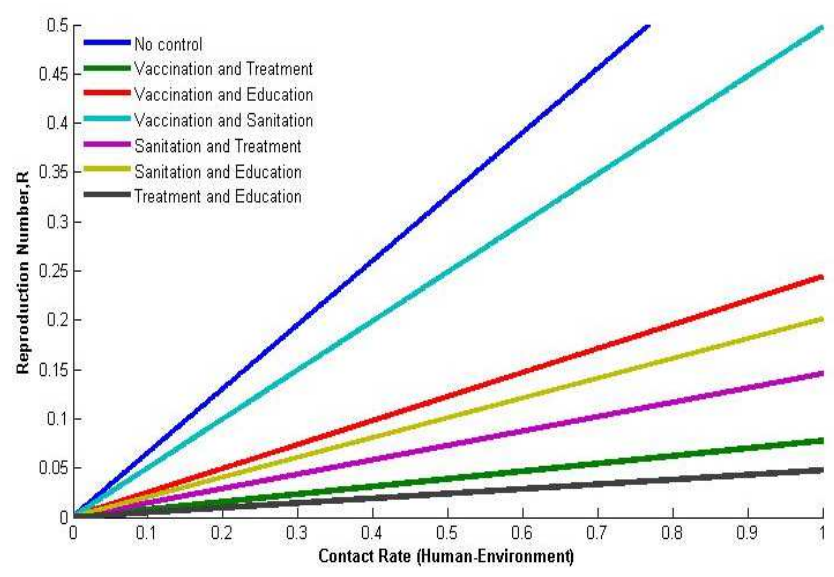

Figure 3. Variations of a bi-control strategies reproduction numbers with respect to human-environment contact rate

Clearly, we observe in Figure 3 that there is a drastic reduction in disease as compared to Figure 2. Our simulations with bi-controls lead to the inequality: $R_{\text {Ted }}<\mathrm{R}_{v T}<R_{s a T}<R_{\text {saed }}<R_{v e d}<R_{\text {sav }}$ as seen from Figure3.
It is obvious that $R_{s a v}$ is worst case scenario, it occurs when a combination of sanitation and vaccination as control strategies to limit the epidemic cholera. It is at the peak, this implies that there is a high increase in reproduction number with respect to human-environment contact rate. Such an increase results in the eruption of cholera in the community.

The best case scenario occurs at graph $\mathrm{R}_{\text {Ted }}$, here two control strategies namely treatment and education are incorporated. The next to best case scenario occurs at graph $\mathrm{R}_{v T}$, in which the combination of vaccination and treatment were considered, followed by $\mathrm{R}_{s a T}$ which is a combination of water sanitation and treatment. Furthermore, we have $\mathrm{R}_{\text {saed }}$, which is a combination of sanitation and education.

From Figure 3, it can be further seen that most of the reproduction numbers are less than 0.25 ; this implies that there is a good control of the disease. Therefore, increasing the number of controls together with their associated parameters values yield a rapid decay of the reproduction number curves. This means that the disease is not endemic and it dies out.

We can therefore conclude that combination of two control strategies is better than one control strategy as it yields better results in diminishing cholera from the community.

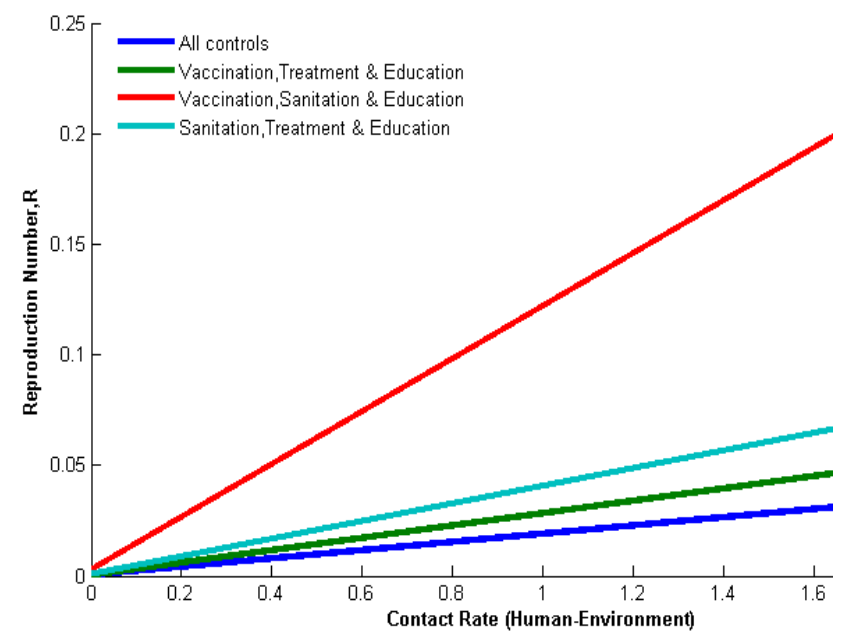

Figure 4.Variations of a tri-control strategies reproduction numbers with respect to human-environment contact rate

Clearly, we observe in Figure 4 that there is a drastic reduction in disease as compared to both Figure 2 and Figure 3 , where most of the reproduction numbers are far less than unity. This implies that there is a control of the disease. Three controls gives results that are marked lower than the results obtained with unique control and a combination of two interventions. Therefore, increasing the number of controls together with their associated parameters values yield a rapid decay of the reproduction number curves. This means that the disease is not endemic and it dies out.

Our simulations with tri-controls lead to the inequality: $R_{\text {all }}<\mathrm{R}_{v \text { Ted }}<R_{\text {saTed }}<R_{\text {vsaed }}$ as seen from Figure 4. It is obvious that $R_{\text {vsaed }}$ is worst case scenario, which is a combination of sanitation, education and vaccination as 
control strategies to limit the epidemic cholera. $R_{v s a e d}$ is at the peak, this implies that there is a high increase in reproduction number with respect to respect to humanenvironment contact rate. Such an increase results in the eruption of cholera in the community.

With regard to the three controls, the best case scenario occurs at graph $\mathrm{R}_{\text {vTed }}$, where three control strategies such as vaccination, treatment and education are incorporated. The next to best case scenario occurs at graph $R_{\text {saTed }}$, in which the combination of sanitation, treatment and education were considered. Furthermore, we have $\mathrm{R}_{\text {ssaed }}$, which is a combination of vaccination, sanitation and education.

From Figure 3, it can be further seen that most of the reproduction numbers are less than 0.25 , which implies that there is a good control of the disease. Therefore, increasing the number of controls together with their associated parameters values yield a rapid decay of the reproduction number curves. This means that the disease is not endemic and it dies out. It is obvious from Figure 4, reproduction number with all four controls, $\mathrm{R}_{\text {all }}$ is the least and of course the best strategy among all controls.

We can therefore conclude that the more you increase combination of control strategies the better you control cholera.

\section{Sensitivity Analysis}

Sensitivity analysis is used to determine how "sensitive" a model is to changes in the value of the parameters of the model and to changes in the structure of the model. Sensitivity analysis helps to build confidence in the model by studying the uncertainties that are often associated with parameters in models. Sensitivity indices allow us to measure the relative change in a state variable when a parameter changes. Sensitivity analysis is commonly used to determine the robustness of model predictions to parameter values (since there are usually errors in data collection and presumed parameter values). Thus we use it to discover parameters that have a high impact on $R_{0}$ and should be targeted by intervention strategies. If the result is negative, then the relationship between the parameters and $R_{0}$ is inversely proportional. In this case, we will take the modulus of the sensitivity index so that we can deduce the size of the effect of changing that parameter. On the other hand, a positive sensitivity index means an increase in the value of a parameter.

The explicit expression of $R_{0}$ is given by the equation (10). Since $\mathrm{R}_{0}$ depends only on six parameters, we derive an analytical expression for its sensitivity to each parameter using the normalized forward sensitivity index as by Chitnis[32] as follows:

$$
\begin{aligned}
& \Upsilon_{k}^{R_{0}}=\frac{\partial R_{0}}{\partial k} \times \frac{k}{R_{0}}=-1 \\
& \Upsilon_{\Lambda}^{R_{0}}=\frac{\partial R_{0}}{\partial \Lambda} \times \frac{\Lambda}{R_{0}}=+1
\end{aligned}
$$

The rest of sensitivity indices for all parameters used in equation (14) can be computed in the similar approach. Table 3 shows the sensitivity indices of $\mathrm{R}_{0}$ with respect to the six parameters.

Table 3. Sensitivity indices of $R_{0}$ with respect to each parameter

\begin{tabular}{ll}
\hline Parameter & Sensitivity Index \\
\hline$\theta$ & +0.0323 \\
$\phi$ & -0.1916 \\
$\alpha_{1}$ & +0.9524 \\
$\alpha_{2}$ & +0.476 \\
$\omega$ & -0.0074 \\
$\gamma$ & -0.8434 \\
$\psi_{e}$ & -1.3016 \\
$k$ & -1 \\
$\Lambda$ & +1 \\
$\mathcal{E}$ & +0.4711 \\
\hline
\end{tabular}

From Table 3, we can obtain $\Upsilon_{k}^{R_{0}}=-1$, this means that an increase in $k$ will cause a decrease of exactly the same proportion in $R_{0}$. Similarly, a decrease in $k$ will cause an increase in $R_{0}$, as they are inversely proportional. We can also note that $\phi$ or $\omega$ or $\psi_{e}$ or $\gamma<0$ hence these parameters are inversely proportional to $R_{0}$.

We also note that ${ }^{\prime} \Upsilon_{\Lambda}^{R_{0}}=+1$, this means that an increase in $\Lambda$ will cause an increase of exactly the same proportion in $R_{0}$. Similarly, a decrease in $\Lambda$ will cause a decrease in $R_{0}$, as they are directly proportional. We can also note that $\theta$ or $\alpha_{1}$ or $\varepsilon$ or $\alpha_{2}>0$ hence these parameters are directly proportional to $R_{0}$.

We can arrange these parameters in the order of their magnitude from the largest to the smallest as follows: $\psi_{e}, \Lambda$, $k, \alpha, \gamma, \varepsilon, \phi, \theta$ and the least sensitive parameter is $\omega$.

Therefore, in order to minimize cholera transmission in a population, this study recommends that, education campaign should be given high emphasis followed by reducing contact rate with contaminated water and food.

The other important issue that follows is treatment; sick people should be quickly given medical attention since the disease kills within in just few hours. Treatment of infected people increases the progression rate to recovered stage and treatment of infectious people will stop them from transmitting the disease.

It can also be noted that there is a need to minimize contact between the susceptible and the infected so as to limit the spread of cholera; it is customary to quarantine the infected individual with the main purpose of minimizing contact rate hence reducing the outbreak of cholera.

It can also be noted that there is a need to minimize contribution of infected people to water and food through fecal contamination. When such contaminated water is drunk by individuals then cholera spreads rapidly in the community.

Another concern that should be given emphasis is vaccination, it is clear that vaccination in many disease has proved to be efficient intervention means, even though most 
vaccines are not $100 \%$ efficient they wane with time. This is due to the fact that, vaccination reduces the likelihood of an individual to be infected.

\section{Conclusions and Recommendations}

\subsection{Conclusions}

In this paper, we have formulated a deterministic mathematical model for transmission dynamics of cholera that incorporates four control strategies namely education, vaccination, treatment, and sanitation. From the model we have derived the effective reproduction number from which we have deduced the basic reproduction number, and the reproduction numbers with combination of two, and three control strategies. The effective reproduction number computed has been used to measure the relative impact for individual or combined intervention for effective disease control. We have derived both the Disease Free Equilibrium (DFE) and the Endemic Equilibrium points (EE) and proved that the DFE is locally asymptotically stable (1.a.s) when $R_{e}<1$.

Moreover, we have performed sensitivity analysis on the basic reproduction with all control strategies, from which we have noted that the most sensitive parameters are the education campaign, therapeutic treatment, effective contact between the susceptible and infected individuals, bacteria carrying capacity, and recruitment rate. These strategies need high attention when at all we need to control cholera outbreak wherever it occurs.

Numerical simulations of the model have shown that, whenever the control strategies are carried out solely then treatment is best alternative to cholera, but when there are two combinations strategies then the best combination is treatment and education.

On the other hand when a combination of three control strategies is implemented then the best combination is the one with vaccination, treatment and education. It has been noted that the best combination is the one that incorporated all four control strategies. From this study we conclude that the more one increases combination of control strategies then cholera is can be eradicated from the community.

\subsection{Recommendations}

Based on our findings, we recommend that proper education and sensitization be given to the public by relevant authorities and NGO's of the dangers of open defecation and urinating in source of drinking water. This will reduce the contribution of each infected person to the aquatic reservoir (parameter $\mathcal{E}$ ).

Also, we recommend that the Government should provide portable water to the populace in order to discourage drinking of untreated water. This will reduce the rate of exposure to contaminated water (parameter $\alpha_{1}$ ). The same was recommended by Ochoche [7].

In addition, it is recommended that people suffering from cholera should be immediately quarantined so as to reduce the contact rate between the infected and the susceptible humans (parameter, $\alpha_{2}$ ). Early treatment of all cholera patients is highly recommended to save the life of the sick humans since cholera kill in very short time (parameter $\gamma$ ). People should be restricted to enter in places where cholera outbreak occurs, this will help to limit the spread of the disease (parameter $\Lambda$ ). In case the economy allows, vaccination strategy should be established to areas where cholera is chronic.

\section{References}

[1] World Health Organization web page: www.who.org

[2] K. Goh, S. Teo, S. Lam, M. Ling. Person-to-person transmission of cholera in a psychiatric hospital. Journal of Infection 20 (1990) 193

[3] H.W. Hethcote, The mathematics of infectious diseases, SIAM Review 42 (2000) 599.

[4] Mukandavire Z, Liao S, Wang J, Gaff H, Smith DL, Morris JG, Estimating the reproductive numbers for the2008-2009 cholera outbreaks in Zimbabwe, Proc Natl Acad Sci 108:8767-8772, 2011.

[5] Tuite AR, Tien J, Eisenberg M, Earn DJ, Ma J, Fisman DN, and Cholera epidemic in Haiti, 2010: Using a transmission model to explain spatial spread of disease and identify optimal control interventions, Ann Intern Med 154:593-601, 2010.

[6] WHO/Cholera Fact 2010.

[7] J.M, Ochoche. A Mathematical Model for the Dynamics of Cholera with Control Strategy. International Journal of Science and Technology, 2013; 2 (11): 797-803.

[8] P.T. Tian, S. Liao, J. Wang. Dynamical analysis and control strategies in modeling cholera. A monograph, 2010.

[9] Hargreaves JR, Bonell CP, Boler T, Boccia D, Birdthistle I, Fletcher A, Pronyk PM, Glynn JR. Systematic review exploring time trends in the association between educational attainment and risk of HIV infection in sub-Saharan Africa. AIDS 22:403-414, 2008.

[10] Barry M, The tail end of Guinea worm Global eradication without a drug or a vaccine. New England J Med 356:2561$2563,2007$.

[11] Smith RJ, Cloutier P, Harrison J, Desforges A: A mathematical model for the eradication of Guinea Worm Disease, in Mushayabasa S (ed.). Understanding the Dynamics of Emerging and Re-emerging Infectious Diseases Using Mathematical Models, pp. 157-177, 2012.

[12] Ramjee G, Gouws E, Andrews A, Myer L, Weber AE. The acceptability of a vaginal microbicide among South African men. Fam Plan Perspect 27:164-170, 2001.

[13] Einarsd'ottir J, Gunnlaugsson G. Health education and cholera in rural Guinea-Bissau. Int J Infect Dis 5:133-138, 2001.

[14] Hubley J, Communicating Health: An Action Guide to Health Education and Health Promotion, MacMillan Press, London, 1993.

[15] Rideal S. Disinfection and Disinfectants (an introduction to the study of).Griffin, London, 1895. 
[16] C. T. Codeço. Endemic and epidemic dynamics of cholera: the role of the aquatic reservoir. BMC Infectious Diseases 1 (2001), 1 .

[17] D. M. Hartley, J. G. Morris Jr. and D. L. Smith. Hyperinfectivity: A critical element in the ability of V. cholerae to cause epidemics? PLoS Medicine 3(2006), 00630069.

[18] D. S. Merrell, S. M. Butler, F. Qadri et al. Host-induced epidemic spread of the cholera bacterium, Nature 417 (2002), 642-645.

[19] S. Liao and J. Wang. Stability analysis and application of a mathematical cholera model. Math. Biosci. Eng. 8 (2011), 733-752.

[20] R. I. Joh, H. Wang, H. Weiss and J. S. Weitz. Dynamics of indirectly transmitted infectious diseases with immunological threshold. Bull. Math. Biol. 71 (2009), 845-862.

[21] Z. Mukandavire, S. Liao, J. Wang, H. Gaff, D. L. Smith and J. G. Morris Jr. Estimating the reproductive numbers for the 2008-2009 cholera outbreaks in Zimbabwe. Proc. Nat. Acad. Sci. 108 (2011), 8767-8772.

[22] J. H. Tien and D. J. D. Earn. Multiple transmission pathways and disease dynamics in a waterborne pathogen model. Bull. Math. Biol. 72 (2010), 1502-1533.

[23] J. Tian and J. Wang. Global stability for cholera epidemic models. Math. Biosci. 232 (2011), 31-41.

[24] R. L. M. Neil an, E. Schaefer, H. Gaff, K. R. Fister and S. Lenhart, Modelingoptimal intervention strategiesfor cholera, Bull. Math. Biol. 72 (2010), 2004-2018.

[25] Diekman, O., Heesterbeek, J.A.P and Metz, J.A.P. On the definition and Computation of the basic reproduction ratio $R_{0}$ in the model of infectious disease in Heterogeneous populations. Journal of Mathematical Biology. 2(1):265382,1990

[26] Van den Driessche, P and Watmough, J. Reproduction numbers and sub-threshold endemic equilibria for compartmental models of disease transmission. Mathematical Biosciences, 2002, 180 (1-2): 29-48.

[27] Stephen, E., Dmitry, K. and Silas, M. Modeling and Stability Analysis for a Varicella Zoster Virus Model with Vaccination. Applied and Computational Mathematics. Vol. 3, No. 4, 2014, pp. 150-162.doi: 10.11648/j.acm.20140304.16
[28] Stephen, E., Dmitry, K., and Silas, M. Modeling the Impact of Immunization on the epidemiology of Varicella-Zoster Virus. Mathematical theory and Modeling.Vol.4, No. 8, 2014, pp.4656 .

[29] N. Nyerere, L. S. Luboobi and Y. Nkansah-Gyekye. Modeling the Effect of Screening and Treatment on the Transmission of Tuberculosis Infections. Mathematical Theory and Modeling. Vol.4, No. 7,2014,pp.51-62.

[30] N. Nyerere, L. S. Luboobi and Y. Nkansah-Gyekye. Bifurcation and Stability analysis of the dynamics of Tuberculosis model incorporating, vaccination, Screening and treatment. Communications in Mathematical biology and Neuroscience, Vol. 2014 (2014), Article ID 3.

[31] J. R. Andrews, S. Basu. Transmission dynamics and control of cholera in Haiti: An epidemic model. Lancet 2011; 377: 124855 .

[32] Chitnis, N., Hyman, J.M., and Cusching, J. M. (2008). Determining important Parameters in the spread of malaria through the sensitivity analysis of a mathematical Model. Bulletin of Mathematical Biology 70 (5):1272-12

[33] Z. Mukandavire, S. Liao, J. Wang, H. Gaff, D. L. Smith and J. G. Morris Jr. Estimating the reproductive numbers for the 2008-2009 cholera outbreaks in Zimbabwe, Proc. Nat. Acad. Sci. 108 (2011), 8767-8772.

[34] D. M. Hartley, J. G. Morris Jr. and D. L. Smith, Hyperinfectivity: A critical element in the ability of V. cholerae to cause epidemics?. PLoS Medicine 3(2006), 00630069 .

[35] Senelani D. Hove-Musekwa, Farai Nyabadzac, Christinah Chiyaka, Prasenjit Das, Agraj Tripathi, Zindoga Mukandavire. Modelling and analysis of the effects of malnutrition in the spread of Cholera. Mathematical and Computer Modelling 53 (2011)1583-1595.

[36] World Health Organization web page: www.who.org.

[37] WHO (2012) http://www.who.int/gho/epidemicdiseases/ cholera/ en/index.

[38] Mwasa, A., Tchuenche, J. M., Mathematical analysis of a cholera model with public health interventions, Biosystems 105:190-200, 2011. 